\title{
Characterization of Chitosan and fabrication of Chitosan hydrogels matrices for biomedical applications
}

\author{
I. Charhouf, A. Benaamara, A. Abourriche and M. Berrada \\ Department of Chemistry, Faculty of sciences Ben M'sik, Casablanca, Morocco
}

\begin{abstract}
The development of injectable hydrogels for drug delivery is a major challenge. Chitosan is a copolymer of Nacetylglucosamine and glucosamine units and is represented as a copolymer. Chitosan occurs in nature in the cell walls of some fungi, exoskeletons of insects and marine animals such as crabs and prawns. Chitosan and its derivatives possess a wide range of useful properties. They are biodegradable, and biocompatible with antibacterial and antioxidant activities. They are useful in drug delivery formulations and tissue engineering. The objective of the present study was to characterize chitosan, prepare chitosan hydrogels and study the gelation of this hydrogels over time. Chitosan with DDA\% 80\%-90\% were characterized by infrared spectroscopy, conductimetry and $\mathrm{pH}$-metry. In addition, chitosan hydrogels were prepared using an ionic gelation method making it suitable for biomedical applications.
\end{abstract}

\section{INTRODUCTION}

During the past decade, novel polymeric microspheres, polymer micelles, and hydrogel-type materials have been shown to be effective in enhancing drug targeting specificity, lowering systemic drug toxicity, improving absorption rates, and providing protection for pharmaceuticals against biochemical degradation. These are all goals of drug delivery $[1,4]$.

Macromolecular drugs are becoming a very important class of therapeutic agents as a result of gaining more understanding of their role in physiology and the rapid advances in the field of biotechnology and genetic engineering. However, naked delivery of these drugs suffered from short half-life, unfavourable pharmacokinetics and systemic toxicity. Therefore, efficient drug delivery systems are necessary to deliver these drugs to achieve the desired therapeutic action. Chitosan-based drug delivery systems are an interesting system for macromolecular drugs $[2,4]$.

Chitosan $[\alpha \quad(1-4)$ 2-amino 2-deoxy $\beta$-D glucan], a copolymer of glucosamine and $\mathrm{N}$-acetylglucosamine (Figure 1), is obtained by deacetylation of chitin, a naturally abundantly available polymer (e.g. in crustaceans). Because of its favourable properties, as discussed in the present article as well as other articles in this issue, chitosan has been studied as a biomaterial and as a pharmaceutical excipient in drug formulations. The primary amine groups introduce special properties that render chitosan very useful for pharmaceutical applications. Because of the presence of functional groups (amine and hydroxyl) various chemical chitosan derivatives have been synthesized and studied for different applications $[3,4]$.

Hydrogels are three-dimensional, hydrophilic, polymeric networks capable of imbibing large amounts of water or biological fluids, and are favored in a broad range of pharmaceutical and biomedical applications. Both natural and synthetic polymers can be used for the production of hydrogels $[5,6]$.

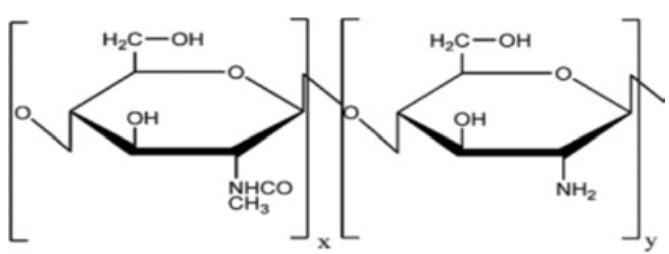

Figure 1. Chemical structure of chitosan.

Crosslinking of the polymer chains can be achieved by various chemical or physical crosslinking methods. Conventional chemically crosslinked hydrogels are often applied as implantables, and the incorporation of drugs by solution sorption may limit the loading level and be time consuming. In addition, the covalent crosslinking reaction may conjugate the drug to the hydrogel or impair the chemical integrity of drugs. The hydrogel also may become non-biodegradable, with ill-defined composition. Therefore, a drug delivery formulation formed by physical hydrogels, where gelation and drug loading can be achieved simultaneously in an aqueous environment without chemical crosslinking, would be attractive [5].

So far, various approaches have been exploited to develop physical polymer hydrogels from different polymers, including gelation in response to temperature or $\mathrm{pH}$ change, hydrophobic interaction, crystallization and ionic interaction, or based on the complexation of enantiomeric polymer or polypeptide segments [1,7].

Admixing a glycerol-phosphate disodium salt to a chitosan aqueous solution, an example of this system, increases the $\mathrm{pH}$ of the solution due to the neutralizing effect of the phosphate groups (base). In the presence of this salt, however, chitosan solutions remain liquid below room temperature, even with $\mathrm{pH}$ values within a physiologically acceptable neutral range from 6.8 to 7.2 . These nearly neutral chitosan/glycerol-phosphate (C/GP) aqueous solutions will gel quickly when heated [7].

The use of complexation between oppositely charged macromolecules to prepare chitosan hydrogels has attracted much attention because the process is very simple 


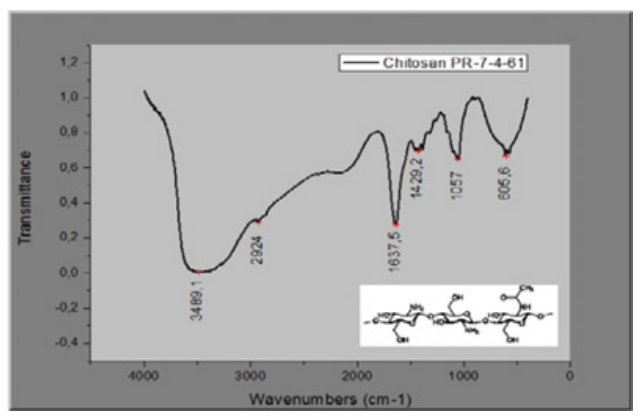

Figure 2. Infrared spectra of Chitosan.

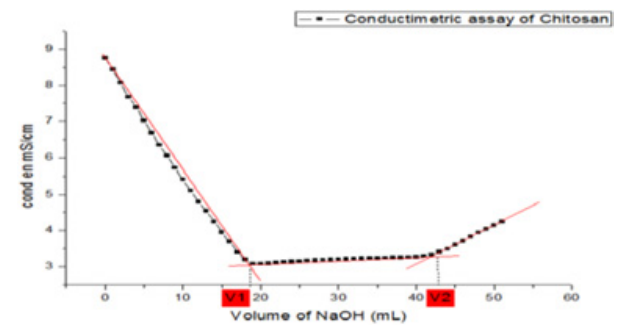

Figure 3. Conductimetric assay of chitosan.

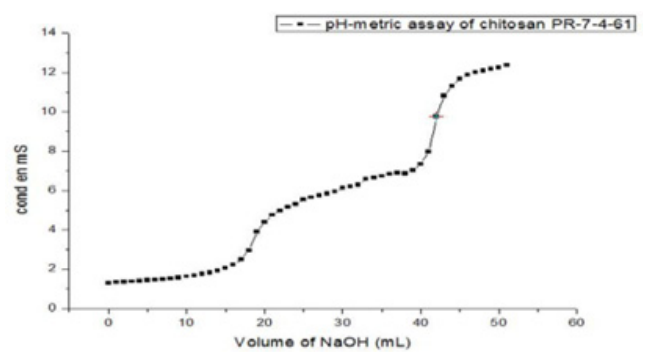

Figure 4. H metric assay of chitosan.

and mild. In addition, reversible physical cross-linking by electrostatic interaction, instead of chemical cross-linking, has been applied to avoid the possible toxicity of reagents and other undesirable effects [8].

\section{MATERIALS AND METHODS}

Chitosan with DDA 80\%-90\% in powder form were prepared at Marinard Biotech Canada (Quebec). One lot of chitosan was used in this study: PR-7-4-61. The disodium $\beta$-Gp (glycerol 2-phosphate disodium salt hydrate were obtained from Sigma Aldrich. Acetic acid, sodium hydroxide and potassium bromide were analytical reagent grade.

Conductimetric and $\mathrm{pH}$ metric measurements were performed with 4310 JENWAY conductivity meter and Eutech pH 510 pH-meter successively.

\section{RESULTS}

\subsection{Structural identification}

The spectrum of chitosane shows a large band at $3100-3500 \mathrm{~cm}^{-1}$ corresponding to stretching vibrations of
$-\mathrm{NH}$ and $-\mathrm{OH}$ bonds including hydrogen bonding. The absorbance peaks due to stretching vibrations of- $\mathrm{CH}$ and -CH2 appear at $2880-2924 \mathrm{~cm}^{-1}$. The characteristic peak appears at $1640 \mathrm{~cm}^{-1}$. It is due to stretching vibrations of the $-\mathrm{CONH} 2$.

\subsection{Calculation of DDA}

\subsubsection{The calculation of DDA}

$$
\mathrm{DDA}=\frac{203 \times\left(\mathrm{V}_{2}-\mathrm{V}_{1}\right) \times \mathrm{N}}{\mathrm{w}+42 \times\left(\mathrm{V}_{2}-\mathrm{V}_{1}\right) \times \mathrm{N}} \times 100
$$

$\mathbf{N}$ : Normality of $\mathrm{NaOH}(\mathrm{mol} / \mathrm{L})$;

V1 and V2: Volumes of $\mathrm{NaOH}$ added;

W: Weight of chitosan (g);

203 $(\mathrm{g} / \mathrm{mol})$ : Molecular weight of acetylated monomer; 42 $(\mathrm{g} / \mathrm{mol})$ : Difference between molecular weights of acetylated monomer and deacetylated monomer.

\subsubsection{Results of conductimetric and $\mathrm{pH}$-metric} techniques

\begin{tabular}{|c|c|c|c|}
\hline \multirow{2}{*}{ Product } & \multicolumn{3}{|c|}{ DDA } \\
\hline & Requirement & Conductometry & pH-metry \\
\hline PR 7-4-61 & $80 \%-90 \%$ & $81,09 \%$ & $81,09 \%$ \\
\hline
\end{tabular}

Reliable and quick techniques that measure acetyl contents of chitosan are needed, conductimetric and $\mathrm{pH}-$ metric titrations are two examples of such techniques. The results determined by these two techniques were similar.

\subsection{Synthesis of dialdehyde starch}

Sodium periodate solution $1 \%(\mathrm{w} / \mathrm{v})$ is adjusted to $\mathrm{pH}$ 3.0 with sulfuric acid. Starch is added under vigorous mechanical stirring. The reaction is kept at room temperature for $30 \mathrm{~min}$. The product is filtered and washed with distilled water. After aceton is used to remove water, the powder is dried under vaccum for $24 \mathrm{~h}$ at room temperature. DAS with unknown aldehyde content are obtained.

\subsection{Gelation of C-SB/DAS solution}

\subsubsection{Operating mode}

A $1 \%(w / v)$ chitosan solution was prepared by stirring powdered chitosan in $0.75 \%(\mathrm{v} / \mathrm{v})$ aqueous acetic acid at room temperature overnight and stored at $4{ }^{\circ} \mathrm{C}$. The final $\mathrm{pH}$ value of the chitosan solution was 5.12. A $1 \mathrm{~g}$ of Sodium bicarbonate was added to the chitosan solution under stirring conditions in an ice bath. The final $\mathrm{pH}$ value of the chitosan/ $\mathrm{NaHCO}_{3}$ solution was 6.9. The solutions were labelled C/SB.

We also prepared a $0.2 \%(\mathrm{w} / \mathrm{v}) \mathrm{C}-\mathrm{SB} / \mathrm{DAS}$ solution, means $10 \mathrm{mg}$ of DAS added to $5 \mathrm{ml} \mathrm{C/SB}$. The solutions were labelled C-SB/DAS. 


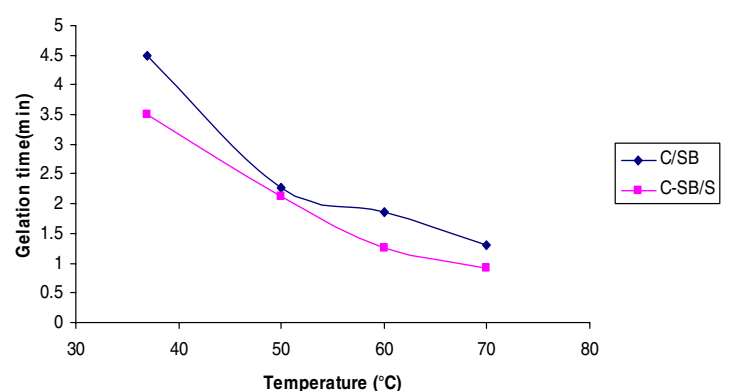

Figure 5. The gelation time of the $\mathrm{C} / \mathrm{SB}$ and $\mathrm{C}-\mathrm{SB} / \mathrm{S}$ solution as a function of the temperature.

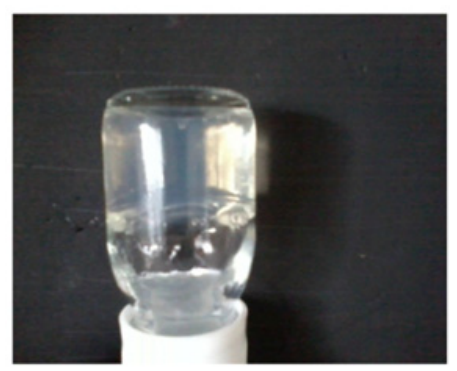

Figure 6. Sample of gel forming C-SB/S.

\subsubsection{Results of gelation time}

In order to investigate the effect of the temperature on the gelation time, the fluidity of chitosan solutions were measured using the test tube inverting method. Figure 5 showed that the gelation time decreased exponentially with increasing temperature. In addition to the changes in flow capabilities, the samples became opaque as the samples formed a gel (figure 6). The C-SB/S showed faster gelation time $\left(3.5 \mathrm{~min}\right.$ at $\left.37^{\circ} \mathrm{C}\right)$ compared with the $\mathrm{C} / \mathrm{SB}(4.5 \mathrm{~min}$ at $37^{\circ} \mathrm{C}$ ). Gel formation times were also different at other temperatures. The shortest gel time observed in this assay was 90 s. [7,9]

\section{CONCLUSIONS}

The addition of a glycerol-phosphate salt to chitosan aqueous solutions directly modulates electrostatic and hydrophobic interactions, and hydrogen bonding between chitosan chains, which are the main molecular forces involved in gel formation. The effective interactions responsible for the sol/gel transition are multiple:

(1) the increase of chitosan interchain hydrogen bonding as a consequence of the reduction of electrostatic repulsion due to the basic action of the salt, (2) the chitosan-glycerol-phosphate electrostatic attractions via the ammonium and the phosphate groups, respectively, (3) the chitosan-chitosan hydrophobic interactions which should be enhanced by the structuring action of glycerol on water.

The nontrivial aspect of such a gelation, namely its temperature dependence, most predominantly originates from the strengthening of chitosan hydrophobic attractions upon increasing the temperature, due to the presence of the glycerol moiety.
At low temperatures, strong chitosan water interactions protect the chitosan chains against aggregation. Upon heating, sheaths of water molecules are removed by the glycerol moiety, which in turn allows association of chitosan macromolecules. Thus, although electrostatic without the attractions through mechanisms (1) and (2), which are present within the C/GP solutions, and which also explain the role of the $\mathrm{pH}$ in the temperature-controlled gelation of C/GP aqueous systems. Thermoreversibility of gelation is linked to the $\mathrm{pH}$ value attained in C/GP solutions before heating and to the fact that cooling after heat-induced gelation weakens hydrophobic forces but strengthens hydrogen bonding.

Thus, systems having $\mathrm{pH}$ value approximately 7.1 before heating appear to be thermoreversible. It should be noted that such a gelation would still not occurs bond formation at lower $\mathrm{pH}$ due to the presence of significant interchain electrostatic repulsion $[7,9]$.

\section{References}

[1] I. Abu Hashim, T. Higashi, T. Anno, K. Motoyama, A. Abd-ElGawad, M. El-Shabouri, T.Borg and H. Arima. Potential use of $\gamma$-cyclodextrin polypseudorotaxane hydrogels as an injectable sustained release system for insulin. International Journal of Pharmaceutics 392, 83-91 (2010).

[2] A. Ahmed, A. Dashevsky and R. Bodmeier. Drug release from and sterilization of in situ cubic phase forming monoglyceride drug delivery systems. European Journal of Pharmaceutics and Biopharmaceutics 75, 375-380 (2010).

[3] M. Amidi, E. Mastrobattista, W. Jiskoot and W.E. Hennink. Chitosan-based delivery systems for protein therapeutics and antigens. Advanced Drug Delivery Reviews 62, 59-82 (2010).

[4] G.Crini, P.M. Badot and E. Guibal. Chitine et Chitosane: Du biopolymère à l'application. Presses universitaires de Franche-Comté.

[5] T. Adali and E. Yilmaz. Synthesis, characterization and biocompatibility studies on chitosan-graft-poly (EGDMA). Carbohydrate Polymers 77, 136-141 (2009).

[6] S.R. Van Tomme, G. Storm and W.E. Hennink. In situ gelling hydrogels for pharmaceutical and biomedical applications. International Journal of Pharmaceutics 355, 1-18 (2008).

[7] A. Chenite, C. Chaput, D. Wang, C. Combes, M.D. Buschmann, C.D. Hoemann, J.C Leroux, B.L. Atkinson, F. Binette and A. Selmani. Novel injectable neutral solutions of chitosan form biodegradable gels in situ. Biomaterials 21, 2155-2161 (2000).

[8] S.A. Agnihotri, N.N. Mallikarjuna, T.M. Aminabhavi. Recent advances on chitosan-based micro- and nanoparticles in drug delivery. Journal of Controlled Release 100, 5-28 (2004).

[9] S. Kim, S. K. Nishimoto, J. D. Bumgardner, W. O. Haggard, M. W Gaber, Y, Yang. A chitosan/bglycerophosphate thermo-sensitive gel for the delivery of ellagic acid for the treatment of brain cancer. Biomaterials 31, 4157-4166 (2010). 